\title{
Modular Disposal Teleoperated Robot
}

\author{
HERNANDO LEON RODRIGUEZ \\ Industrial Engineering Department Faculty Engineering, Nueva Granada Military University, \\ Bogotá - Colombia, hernando.leon@unimilitar.edu.co \\ TARIQ SATTAR \\ Department of Engineering and Design, \\ London South Bank University, 103 Borough Road, SE1 OAA, London - UK \\ sattartp@lsbu.ac.uk
}

\begin{abstract}
The Modular Disposal Teleoperated robot (MDT Robot) can replace man to recognise, and remove explosive bombs or other unknown articles in a dangerous environment. The design is modular, with modules comprising of a platform mobile vehicle, an articulated mechanical arm, a teleoperated control system, vision system and a wireless communications system. The MDT Robot is developed to provide high manoeuvrability and strong capacity to defeat obstacles, stairs, etc. It can be used in urban areas and in wild environments of sand, grass and soft soil, etc. The robot design uses several commonly available recycled industrial parts to produce a very low cost disposable robot whose loss can be easily accepted.
\end{abstract}

Keywords -MDT Robot, mobile vehicle, articulated mechanical arm, teleoperated control system, vision system.

\section{Introduction}

A disposal robot is an unmanned machine operated remotely with the intention of removing or making safe an unknown suspect package. Disposal teleoperated robots are the focus of military research. These robots are required to achieve complex missions and sometime deal with complicated explosive devices. Most of these robots are teleoperated machines that can recognise, dispose, move, climb, search, lift and handle dangerous explosives [1] [2].

There are many tasks that are hazardous to human life which could be conducted remotely using teleoperated robots. The main idea is to design simple machines that an operator uses with the help of multiple cameras to obtain different views of any unknown artefact from a safe distance. Currently, a teleoperated robot is completely operated in manual mode and requires constant human intervention, such as image interpretation, manoeuvring the robot from one place to another and object manipulation. In the future, most robots will be fully autonomous to carry out complex operations in shapeless environments without human intervention [3].

The research into EOD (explosive ordnance disposal) robots began in the 1960s with the global situation of terrorist attacks and has been increasingly used in war. These robots have used stereoscopic vision systems to perform inspection, grasp and destroy tasks [1] [4].

A large number of companies, universities and research institutions are working on EOD robots. Some of them are in the United States: Remotec, irobot, Wolstenholme, In the United Kingdom: ABP, Allen, QinetiQ; in France: Cybernetics, DM Corporation; in Canada: Inuktun, Pedsco; in China: Beijing University of Aeronautics and Astronautics and in Germany: Telerob. etc. [1] [2] [5].

An EOD robot requires strong mechanical design to provide it the ability to operate in various terrains such as roads, fields, and deal with obstructions, stairs, etc. It needs to be light weight to increase speed and operate in rough terrain, be operated easily with a good 
amount of autonomy and be easily repairable. Due to the complex manipulation of the robot and to avoid mistakes or mix ups in pressing control buttons, the operator needs integrated systems to be aware of all data being provided by the robot such as on-site video signals or feedback commands from the robot, etc.

This paper describes the development of a modular disposal teleoperated robot, figure 1 , using recycled parts with the purpose of constructing a basic device composed of a mobile crawler platform, a 5 degree of freedom manipulator arm, wireless communications and a vision system. This robot has been developed with a very low cost budget with the aim of constructing an expendable machine which if destroyed or lost is financially acceptable and the deactivation team can even have a kamikaze machine to save lives at the expense of the robot.

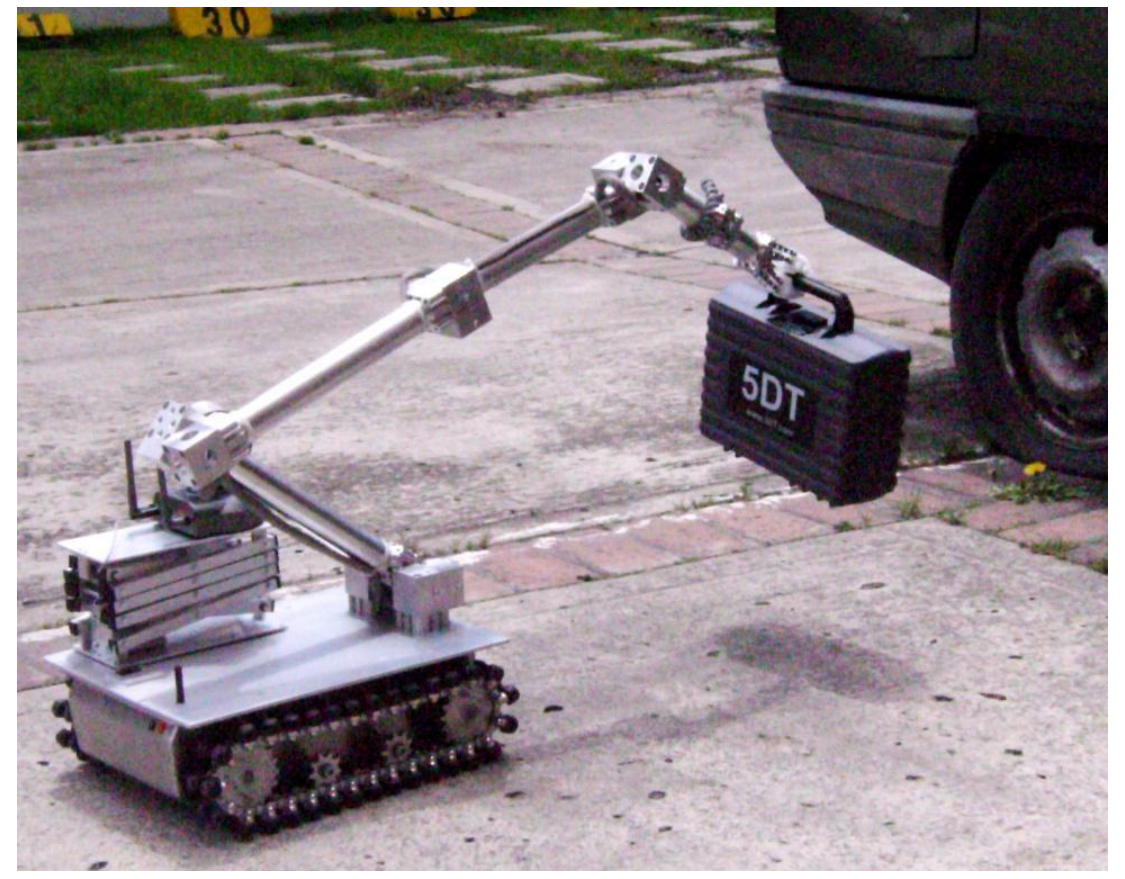

Figure 1: Prototype of modular disposal teleoperated (MDT) robot.

\section{The Modular Disposal Teleoperated Robot}

The MDT robot is composed by four main parts (see figure 2.), the mobile platform, the manipulator arm, the camera scissor lift mechanism and the control and vision system. The mobile platform is a robot vehicle which has 4 driver motors controlled by the one dual motor controller. These motors are mounted in the main structure and provide the linear, rotational and climbing motion capacity of the system. The manipulator arm is composed of a number of similar modules, where links and joints are attached serially and mounted on top of the robot platform. The manipulator contains 5 revolute joints and one gripper placed at its end, which allows it to grasp any object of maximum $60 \mathrm{~mm}$ width. It is controlled by 3 dual motor controllers and provides the possibility to use the Denavit-Hartenberg algorithm kinematics model to move the arm [6] [7]. The main controller and the operator PC/Laptop have a client-server relationship via a graphics user interface (GUI). The operator can access and control wirelessly the mobile platform, the manipulator arm, the camera scissor lift mechanics and the camera itself. The GUI makes the connections straight forward with the main motor controllers, permitting braking, motion control and adjustment of the 
camera by individual control of each joint and also obtains error signals from the 9 motors and their corresponding servo drives.

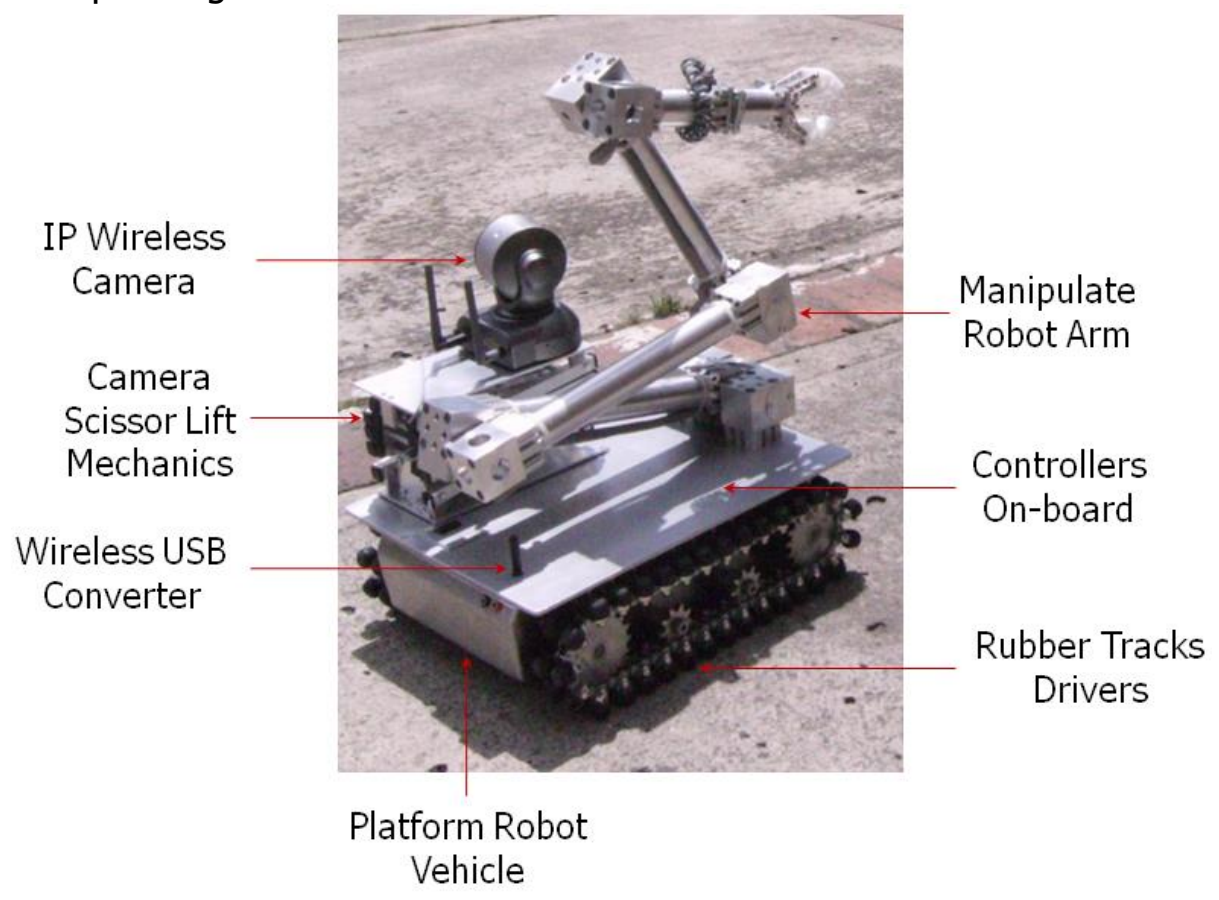

Figure 2: Main parts of the modular disposal teleoperated (MDT) robot.

\section{Mobile Platform}

The mobile platform of EOD robots are mostly wheeled units, tracked units, or a hybrid of these. This project has developed a tracked robot which has an advantage especially in climbing stairs and crossing mixed terrain environments with high friction contact with the surface but has the weakness of a heavy weight structure.

The platform vehicle is showing in figure 3 ; it is a teleoperated mobile vehicle with rubber tracks to drive it. These tracks are basically a mixture of steal chain transmission and rubber caps fixed together to increase surface friction and reduce vibrations.

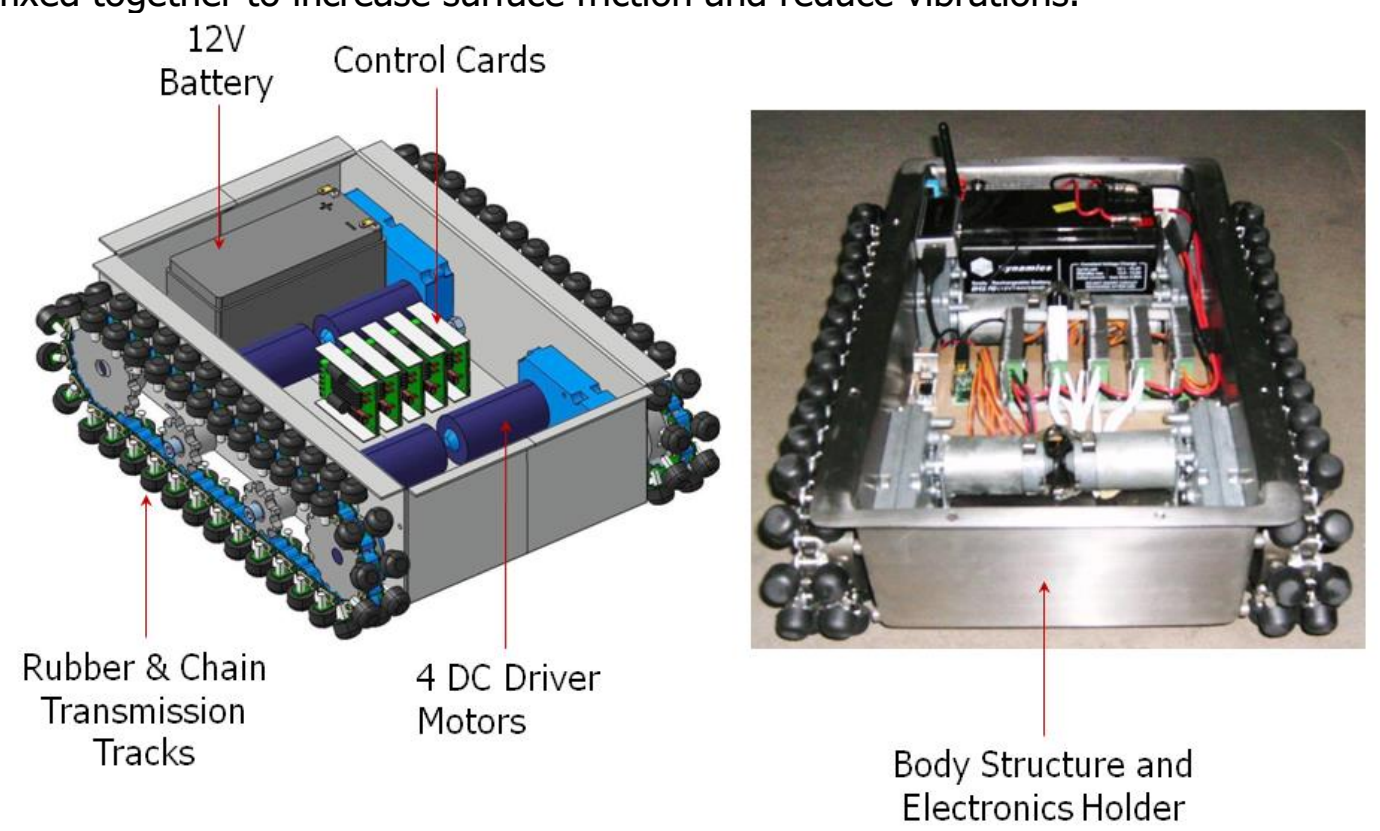


Figure 3: Mobile teleoperated robot platform

The platform vehicle weighs $13 \mathrm{~kg}$ and is composed of a body structure, four driven motors, two flexible steel chain tracks, four driven pinions and four supported pinions. The body structure was made with stainless steel material designed to be light with reduced machining requirements, small size of the vehicle and also strong enough to support the driven motors placed on the sides of the vehicle.

The platform vehicle uses a dual crawler differential drive; each track is driven by two pinions attached to one geared motor with the intention of increasing power to the vehicle. The drive scheme is very simple, reduces mechanical design and control systems and achieves forward, backward, turn left, and turn right motion, climb stairs, climb over obstacles but motion functionality is not very accurate. But for this application it is sufficient to get close to the suspicious object. Since each track has two motors for increased power transmission, both motors are synchronised to avoid one track moving faster than the other.

All electronic cards, batteries and USB wireless devices are placed on-board with the advantage of increasing the mass and hence the vehicle grip to give it enough drive force and stability.

On top of the platform are mounted a manipulator arm to handle the unknown package and a camera scissor lift mechanism to raise/lower the vision system. Both arms can be controlled by the operator and they can be deployed when necessary to grasp any object and/or change the vision angle.

\section{The Manipulator Arm}

Figure 4 shows the manipulator arm of the disposal system. It has been developed in modular principle to provide a great deal of flexibility to reach a given point inside its workspace to collect any unknown object over possible obstacles. The arm is composed of four similar modules on each revolute joint, one rotated joint and one interchangeable gripper. It has redundant joints; its weight is $6 \mathrm{~kg}$ including driven motors. Its structure is totally made in profile aluminium; its frames have been made from modified recycled pneumatic cylinders; normally these actuators are thrown away after being used in automated machines or used as support structures.

These frames cylinders are extremely light and strong and can be very easy replaced in case of failure or totally damaged. The frames are united by the module joints made in aluminium to keep the arm as light as possible, and also these modules are mechanically similar to reduce the cost of the arm and simplify its construction and maintenance. In its fully extended position the manipulator arm can reach forward about 1.2 metre and 1.5 metres high, See figure 4 . It has the capability to carry $2 \mathrm{~kg}$ in its gripper.
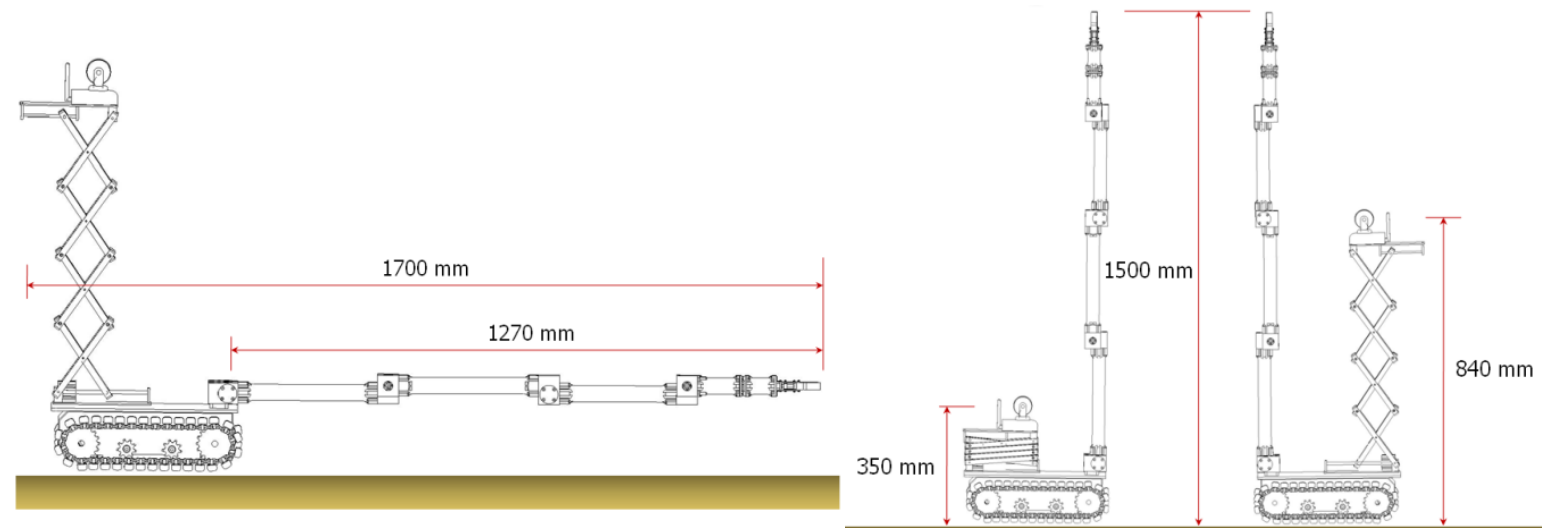
Figure 4: The 5DOF robot arm fully extended.

The manipulator arm is driven with 5 DC motors which are positioned inside each frame and they are controlled independently. The description of the developed arm can be displayed using the following parameters of Denavit-Hartenberg algorithm kinematics model. This specific kinematics model for the manipulator represents the transformation from the base coordinate to the gripper coordinate. The result is a matrix, which transforms the first coordinate into the last coordinate by the settings of the joint angles. See figure 5.

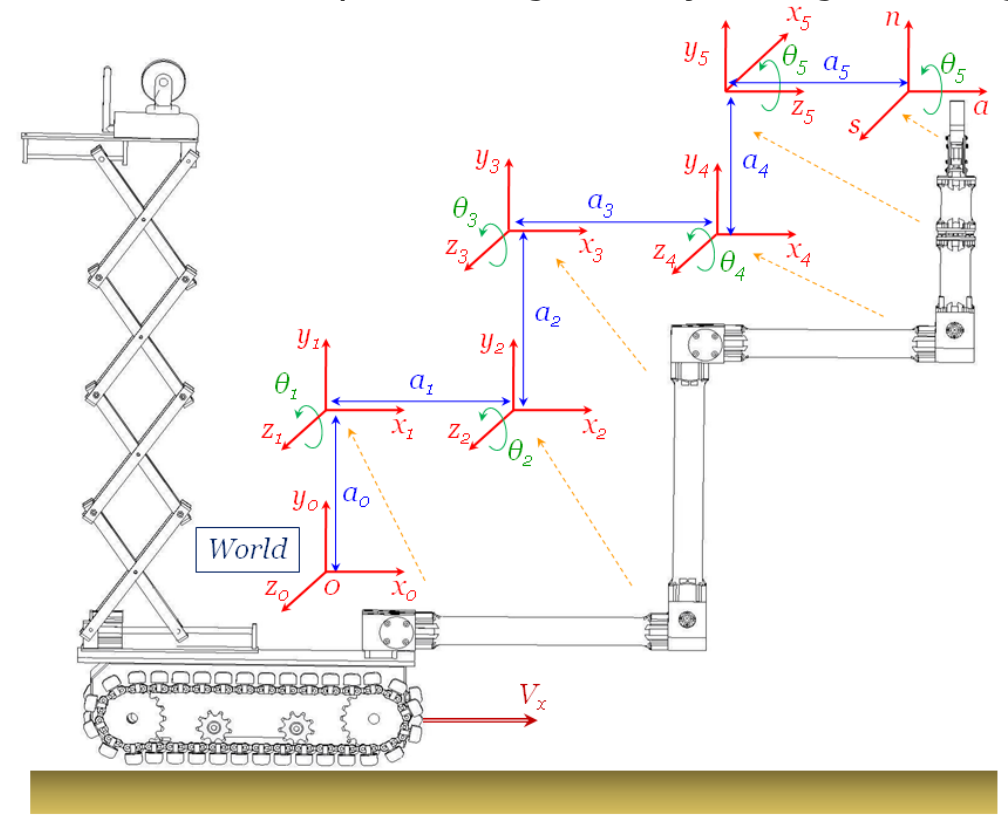

Figure 5: The five degree of freedom kinematics model manipulator arm.

Table 1 shows the parameters and the characteristic representation of the arm and consists of two joint parameters and two link parameters. For an articulated robot whose joints are revolute the joint angle $\left(\theta_{n}\right)$ is the only variable parameter. The joint distance $\left(\boldsymbol{a}_{n}\right)$, the link length $\left(\boldsymbol{d}_{\boldsymbol{n}}\right)$ and the link twist angle $\left(\boldsymbol{\alpha}_{\boldsymbol{n}}\right)$ are fixed parameters.

The link twist angle $\alpha$ is the rotation, which is necessary to transform $z_{n-1}$ to $z_{n}$ by rotating about $\mathrm{x}_{\mathrm{n}}$. And the joint angle $\theta$ is the rotation around the $\mathrm{z}$-axis at will to match the $\mathrm{x}$-axis of the previous coordinate frame $x_{n-1}$ with the one of the following frame $x_{n}$.

Table 1: The resulting characteristic table for the revolute manipulate arm.

\begin{tabular}{||c|c|c|c|c||}
\hline \multirow{2}{*}{ Axis (Joint) } & \multicolumn{2}{|c|}{ Joint } & \multicolumn{2}{c||}{ Link } \\
\cline { 2 - 5 } & $\begin{array}{c}\text { Angle }\left(\theta_{n}\right) \\
\text { - variable - }\end{array}$ & $\begin{array}{c}\text { Distance }\left(a_{n}\right)[\mathrm{mm}] \\
\text { - fixed - }\end{array}$ & $\begin{array}{c}\text { Length }\left(d_{n}\right)[\mathrm{mm}] \\
\text { - fixed - }\end{array}$ & $\begin{array}{c}\text { Twist angle }\left(\alpha_{n}\right) \\
\text { - fixed - }\end{array}$ \\
\hline 1 (Base) & $\theta_{1}$ & 345 & 0 & 0 \\
\hline 2 (Shoulder) & $\theta_{2}$ & 345 & 0 & 0 \\
\hline 3 (Elbow) & $\theta_{3}$ & 310 & 0 & 0 \\
\hline 4 (Wrist) & $\theta_{4}$ & 90 & 0 & $\pi / 2$ \\
\hline 5 (Roll) & $\theta_{5}$ & 0 & 0 & 0 \\
\hline \hline
\end{tabular}


The global world coordinate is assigned to the base so that the z-axis represents the axis of rotation. The z-axes are set to align it with the axis of rotation. The $x$-axes are selected so that they are orthogonal to $\mathrm{z}_{\mathrm{n}-1}$ and $\mathrm{z}_{\mathrm{n}}$. Because the $\mathrm{z}$-axis and $\mathrm{x}$-axis of the coordinate frame are determined, then the $y$-axis is defined. The last coordinate frame is set at the gripper tool tip. The origins of the other coordinate frames are located in the point of intersection of the z-axis, so the joint 1, 2, 3, 4 are parallels with the z-axis but joint 5 rotated 90 degrees.

A commanded position of the robot's gripper generates the control system to make use of the kinematics matrix and determine the right joint angles of all five joints in order to achieve the demanded orientation and position of the robotic arm. The position control system drives each motor optimally to reach a precise placement of each joint. These commands can be run manually or can be a predetermined task already set in the software.

\section{The Control and Vision System}

Figure 6 shows a block diagram of the control system architecture for 9 DC servomotors connected by pair to the dual motor controller. It comprises of the main control, graphic user interface and vision system (software) and several on-board control cards (hardware). The control cards are dual motor driver controllers composed of five DC servomotor cards and embedded amplifiers. The control is PID, running independently for each axis, all cards are connected to the main driver control card. The system can control velocity and position of each motor by sending PWM signals through the main driver controller. These signals are sent by an USB adapter which communicates with the main control wirelessly.

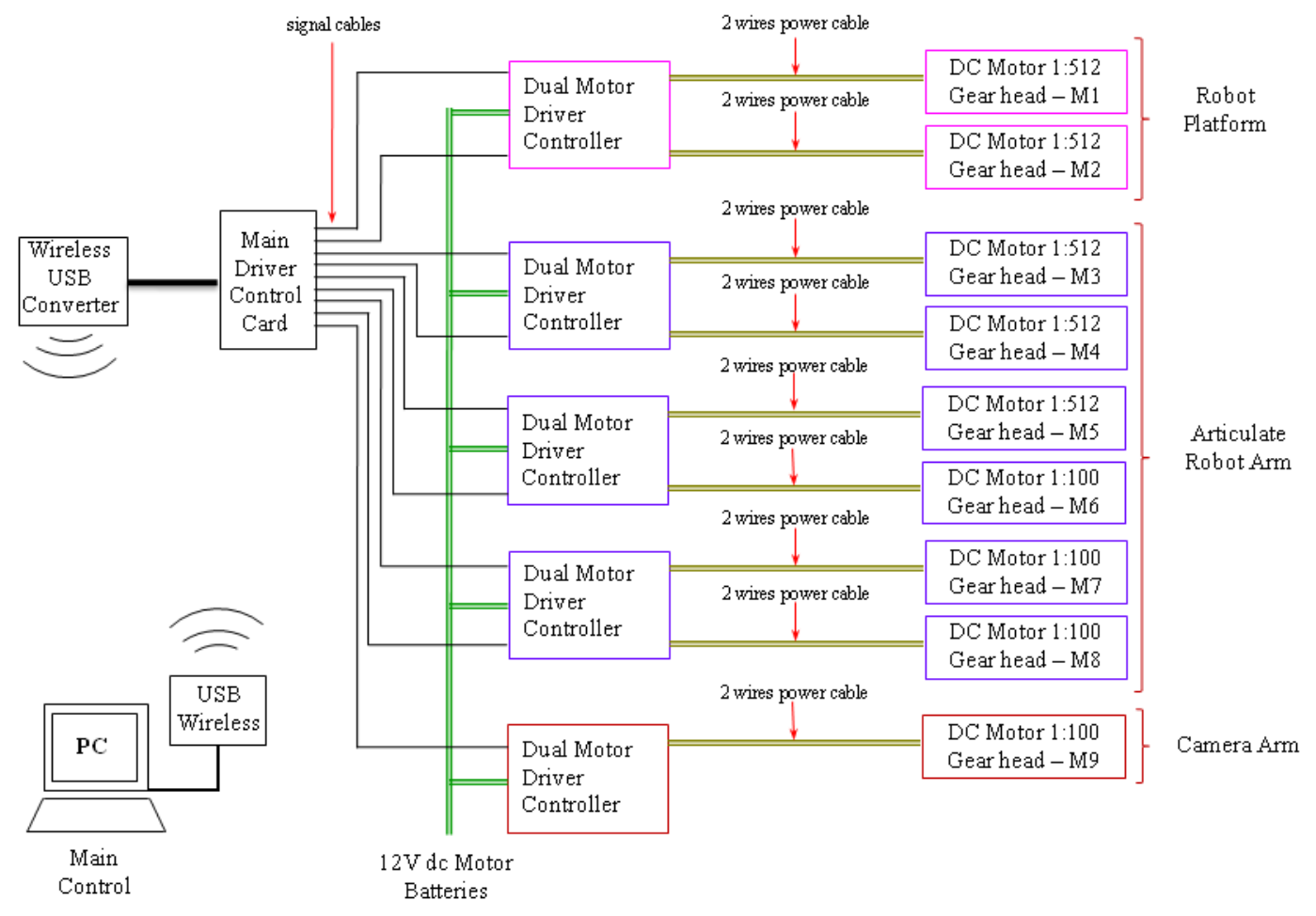

Figure 6: Diagram of the control system of the modular disposal teleoperated robot. 
The control system has been divided to use 9 servo DC motors, which have been organized to control the left track, right track of the mobile platform; base joint motor, shoulder joint motor, elbow joint motor, wrist joint motor, roll joint motor and gripper drive motor for the manipulator arm; and one additional dual control cards for the camera scissor lift mechanics. The data transmission from the on-board control cards is sent by USB wireless communication but the video picture between main computer and the robotic system is sent by Ethernet wireless communications.

An operator gets information about the robot through the remote control terminal, see figure 7, and operates the robot's movements and actions. Control data and the images are transmitted by a separate wireless transmission module.

The interface has been programmed in $\mathrm{C}++$. It has graphic user interfaces (GUI) which control the mobile platform, the manipulator arm, camera scissor lift mechanics video system.

Various control commands are created by combining a number of separate buttons and are divided into the control commands as follows: platform backwards/forward, turn left/turn right and stop movements, up/down/stop movements of each joint in the manipulator arm, up/down camera lift mechanics, video system. The operator can modify manually the speed independently of the platform and the manipulator arm.

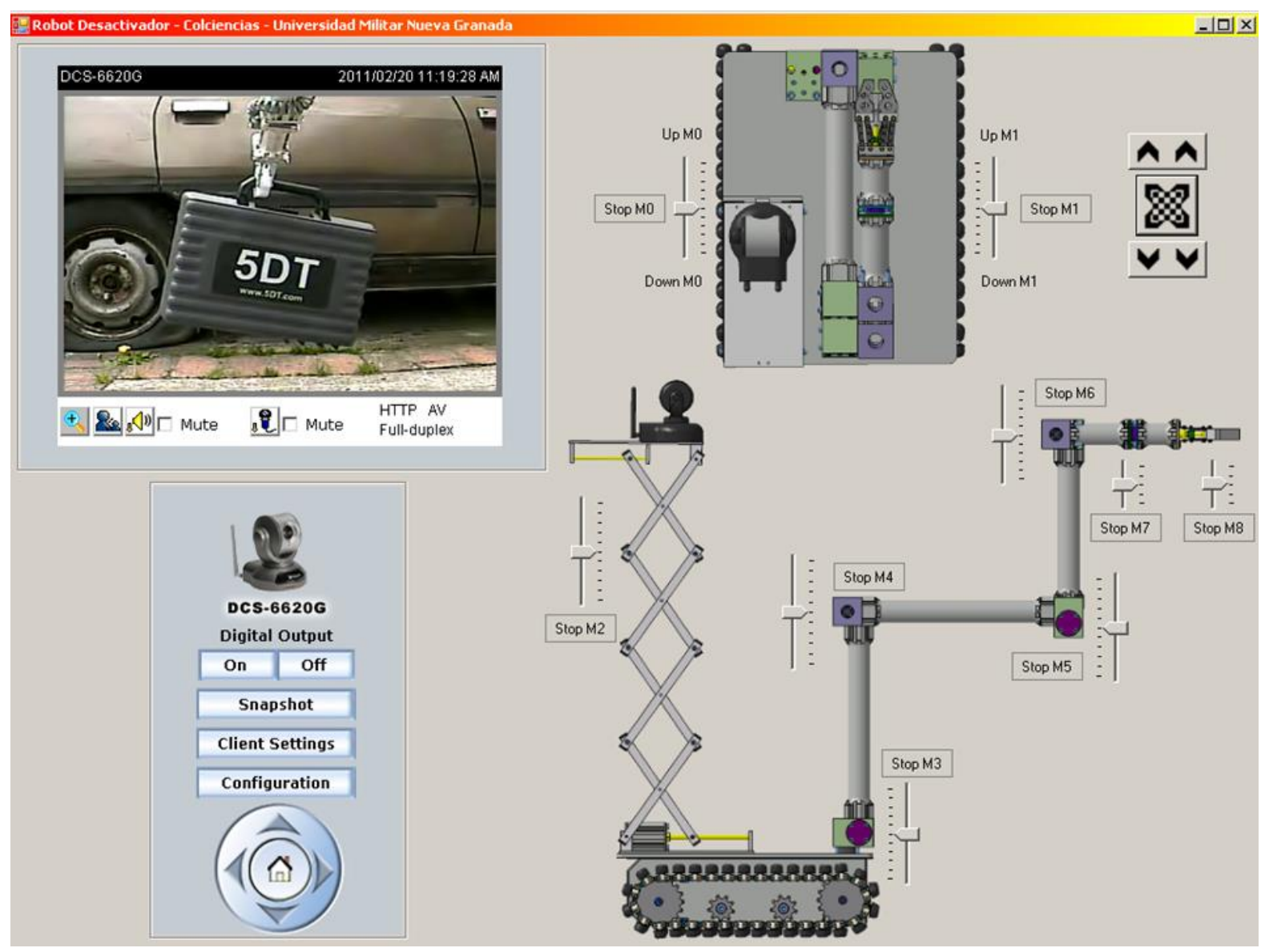

Figure 7: Graphic user interface of the modular disposal teleoperated robot.

The video cameras tilt/pan/zoom is controlled manually by wireless commands and images are displayed in a single screen picture. It can also send/receive audio from the robot device and other features of the camera e.g. I/O command and darkness. The operator can view 
the robot's movements, including speed, direction and manoeuvre the joint angles of the manipulator arm.

For safety, the operator needs to be a least 200 meters away from the robot and preferably 500 meters away from the unknown package. The range of the wireless communications system has been increased to around 300 meter with amplification antenna. In addition the system has 100 metres cables for backup communications purposes.

\section{FUTURE WORK}

The prototype robot system, figure 8 , has being tested on different surfaces and environments but requires more elaborate trials to determine the maximum distance that the system can operate remotely, its accuracy and repeatability. While the camera has performed very well in initial trials, more cameras are needed to increase the view of the operator to give a clear view of the robot and its surroundings to improve situational awareness.

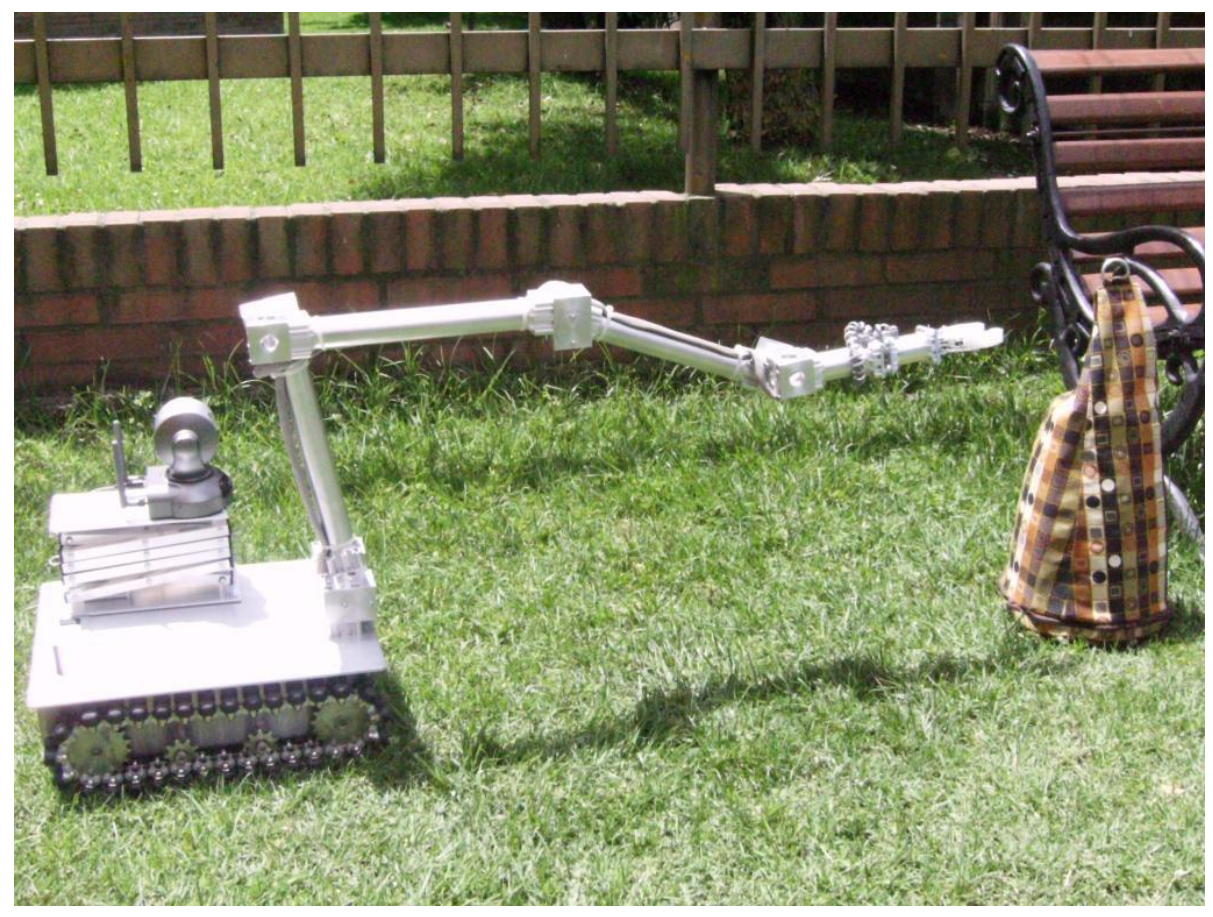

Figure 8: Modular disposal teleoperated robot trial.

\section{Conclusions}

The project has achieved a modular design with recycled materials in the manipulator structure to keep its cost low. The robot has sufficient functionality to do the required tasks and is cheap enough to be easily expendable and lightweight enough to be easily transported and deployed.

It is compact, enabling operation inside buildings, sewers, courtyards and constrained spaces. While intended primarily for explosive ordnance disposal (EOD) it is also suitable for perimeter security using on-board motion and sound detectors and for other tasks such as inspection. 
The robot design proves that a modular robot can be developed with the purpose of reducing cost but yet having high enough capacity to realise complex tasks, cross obstacles and dispose bombs.

\section{ACKNOWLEDGMENT}

The authors would like to thank the national research council of Colombia Colciencias for sponsored this project.

\section{REFERENCES}

[1] Boyu Wei, Junyao Gao, Kejie Li, "Design of a Large Explosive Ordnance Disposal Robot", Second International conference on intelligent computation techonology and automation, pp. 403-406, 2009.

[2] Gao Junyao, Gao Xueshan, Zhu Jianguo, Zhu Wei, Wei Boyu, Wang Shilin, "Heavy Explosive Removing Robot Control Technique Research" International conferences on intelligent human machines systems and cybernetics, pp. 85-89, 2009

[3] Nuttaka Hounsup, terapass Jariyanorawiss, Wiroj Homsup, "A control of A bomb Disposal Robot using a stereoscopic Vision" Ieee, pp. 293-294, 2008

[4] Noel Sharkey, "Cassandra or false prophet of domm: al robot and war", IEEE intelligent Systems, pp. 14-17, 2008

[5] Zhou Hongfu, Xiao Xinyan, Jiang Liangzhong, "DC servo controlled system design on Bomb-Disposal robot algorithm", the eight international Conference on Electronics Mesurement and instruments, pp. 510-514, 2007

[6] Schilling, R. J.: Fundamentals of Robotic 1990, Prentice-Hall Inc, Mexico, USA

[7] Alina-A Brenner, T. P. Sattar (2006), Portable 7 DOF Scanning Arm For Non-destructive Inspection Of Objects With Unknown Geometry Proceedings of the 9th International Conference on Climbing and Walking Robots and the Support Technologies for Mobile Machines, September 12 - 14, 2006, Brussels, Belgium, pp 527-532

[8] Karlin,S. " Mom vs. Bomb". Spectrum, IEEE Volume 45, March 2007 